18. Aparicio, O. M., Billington, B. L. \& Gottschling, D. E. Modifiers of position effect are shared between telomeric and silent mating-type loci in S. cerevisiae. Cell 66, 1279-1287 (1991).

19. Moazed, D. \& Johnson, D. A deubiquitinating enzyme interacts with SIR4 and regulates silencing in S. cerevisiae. Cell 86, 667-677 (1996).

20. Moretti, P., Freeman, K., Coodly, L. \& Shore, D. Evidence that a complex of SIR proteins interacts with the silencer and telomere-binding protein RAP1. Genes Dev. 8, 2257-2269 (1994).

21. Palladino, F. et al. SIR3 and SIR4 proteins are required for the positioning and integrity of yeast telomeres. Cell 75, 543-555 (1993).

22. Porter, S. E., Greenwell, P. W., Ritchie, K. B. \& Petes, T. D. The DNA-binding protein Hdflp (a putative $\mathrm{Ku}$ homologue) is required for maintaining normal telomere length in Saccharomyces cerevisiae. Nucleic Acids Res. 24, 582-585 (1996).

23. Sussel, L. \& Shore, D. Separation of transcriptional activation and silencing functions of the RAP1 encoded repressor/activator protein 1: isolation of viable mutants affecting both silencing and telomere length. Proc. Natl Acad. Sci. USA 88, 7749-7753 (1991).

24. Buck, S. W. \& Shore, D. Action of a RAP1 carboxy-terminal silencing domain reveals an underlying competition between HMR and telomeres in yeast. Genes Dev. 9, 370-384 (1995).

25. Rothstein, R. J. One-step gene disruption in yeast. Methods Enzymol. 101, 202-211 (1983).

26. Thomas, B. J. \& Rothstein, R. Elevated recombination rates in transcriptionally active DNA. Cell 56, 619-630 (1989).

27. Hollenberg, S. M., Sternglanz, R., Cheng, P. F. \& Weintraub, H. Identification of a new family of tissuespecific basic helix-loop-helix proteins with a two-hybrid system. Mol. Cell. Biol. 15, 3813-3822 (1995).

28. Rose, M. D., Winston, F. \& Hieter, P. Methods in Yeast Genetics, a Laboratory Course Manual (Cold Spring Harbor Laboratory Press, NY, 1990).

29. Luria, S. E. \& Delbruck, M. Mutations of bacteria from virus sensitivity to virus resistance. Genetics $\mathbf{2 8}$, 491-511 (1943)

30. Lea, D. E. \& Coulson, C. A. The distribution of the numbers of mutants in bacterial populations. J. Genet. 49, 264-285 (1948).

Acknowledgements. We thank S. Fields, S. M. Hollenberg, K. Johzuka, I. Kobayashi, A. Miyajima, J. Rine and J. W. Szostak for providing plasmids and strains, and K. Johzuka for advice on the two-hybrid assay. This work was supported in part by grants to Y.T., J.K. and H.I. from the Ministry of Education, Science, Sports, and Culture of Japan. Y.T. was supported by a postdoctoral fellowship of the Japan Society for the Promotion of Science.

Correspondence and requests for materials should be addressed to H.I. (e-mail: ike@hgc.ims.utokyo.ac.jp).

\section{Crystal structure of the} breakage-reunion domain of DNA gyrase

\section{João H. Morais Cabral, Andrew P. Jackson, Clare V. Smith, Nita Shikotra, Anthony Maxwell \& Robert C. Liddington}

Department of Biochemistry, University of Leicester, Leicester LE1 7RH, UK

DNA gyrase is a type II DNA topoisomerase from bacteria that introduces supercoils into $\mathrm{DNA}^{1,2}$. It catalyses the breakage of a DNA duplex (the $G$ segment), the passage of another segment (the $T$ segment) through the break, and then the reunification of the break. This activity involves the opening and closing of a series of molecular 'gates' which is coupled to ATP hydrolysis. Here we present the crystal structure of the 'breakage-reunion' domain of the gyrase at $2.8 \AA$ resolution. Comparison of the structure of this $59 \mathrm{~K}$ (relative molecular mass, 59,000) domain with that of a $92 \mathrm{~K}$ fragment of yeast topoisomerase II (ref. 3 ) reveals a very different quaternary organization, and we propose that the two structures represent two principal conformations that participate in the enzymatic pathway. The gyrase structure reveals a new dimer contact with a grooved concave surface for binding the $G$ segment and a cluster of conserved charged residues surrounding the active-site tyrosines. It also shows how breakage of the G segment can occur and, together with the topoisomerase II structure, suggests a pathway by which the $T$ segment can be released through the second gate of the enzyme. Mutations that confer resistance to the quinolone antibacterial agents cluster at the new dimer interface, indicating how these drugs might interact with the gyrase-DNA complex.

An alignment of domains in Escherichia coli gyrase and yeast topoisomerase II (topo II) is shown in Fig. 1, and their threedimensional structures are compared in Fig. 2. GyrA59 is the minimal fragment of the A-subunit which, when complexed with the B-subunit, has DNA-cleavage activity ${ }^{4}$. The carboxy-terminal domain of GyrA is required for the introduction of DNA supercoils, but the GyrA59-GyrB complex supports ATP-dependent relaxation of supercoils in a reaction reminiscent of conventional type II topoisomerases such as yeast topo II (ref. 5).

Each GyrA59 monomer is composed of an N-proximal head and a C-proximal tail. The head contains two domains: one similar to the DNA-binding domain of the catabolite-activator protein (CAP), including the helix-turn-helix (HTH) motif; and a second domain with $\alpha / \beta$ structure (the 'tower' domain) which stands on the CAP-like domain. The tail has a single domain with a helical core. Two long helices $(\alpha 14$ and $\alpha 18)$ emanate from this core and connect, together with the C-terminal helix $(\alpha 19)$, the head and tail fragments. The head fragment has a very similar structure to yeast topo II (ref. 3). The tail is also structurally conserved at its core, although large surface loops emanating from different points give it a different outward appearance. The tail fragments form the so-called 'primary' dimer interface (total buried surface, $2,150 \AA^{2}$ ), which is closely conserved in topo II (refs 3,6 ), providing a convenient reference point from which to compare the two structures.

The three connecting helices $(\alpha 14, \alpha 18$ and $\alpha 19)$ adopt very different conformations, leading to large quaternary movements involving a single hinge-point within the helices and rigid body movements of the head fragments (Fig. 2d). Compared with the yeast structure, the angle between $\alpha 18$ and $\alpha 19$ reduces from $120^{\circ}$ to $90^{\circ}$. At the same time, the long helix $\alpha 14$ bends by $30^{\circ}$ towards the dimer-related molecules and changes the way it packs against $\alpha 18$ and $\alpha 19$, shifting two helical turns on $\alpha 19$. The head fragments, which are rigidly connected to $\alpha 14$, swing towards each other and dock together to form a new contact (the 'head' dimer interface), with the active-site tyrosines moving by $26 \AA$ towards and past each other (Fig. 2a, b). The resultant structure is topologically a closed ring, with external dimensions of $100 \AA \times 40 \AA \times 100 \AA$ and a central hole of diameter $30 \AA$. The hole is much smaller than in the topo II structure but it is still large enough to accommodate a DNA duplex.

The 'head' dimer interface is dominated by an antiparallel sideby-side packing of the $\alpha 3$ helices (the first helix of the HTH motif) from each monomer, together with their adjoining loops (Fig. 3b, c). The helices pack very closely (mean centre-to-centre distance $7 \AA$ ) owing to a preponderance of small side chains. The two abutting surfaces are flat and hydrophobic at the centre, with charged groups at the periphery, burying a total surface area of $1,380 \AA^{2}$. At the top of the interface, the 'recognition' helices $(\alpha 4)$ make a head-to-head antiparallel dimer contact. Dimer formation creates a large concave surface $(100 \AA \times 30 \AA)$ on the top of the molecule formed by the upper surface of the CAP-like domains and the sides of the two 'tower' domains (Fig. 3a). The active-site tyrosines (Tyr 122) are on loops at either end of the dimer interface, $30 \AA$ apart (very similar to their separation in the yeast topo II structure), and sit at the ends of strongly basic grooves created by the dimer-related monomers. Previous biochemical studies have shown that the cleavage of DNA is achieved by a transesterification reaction between the tyrosines and the 'target' phosphoryl groups on opposing strands of the DNA backbone, resulting in the tyrosine being covalently attached to the $5^{\prime}$ end of the cleaved segment with a 4-base overhang ${ }^{1,2}$. The gyrase structure reveals a new cluster of conserved residues (Fig. 3c), juxtaposing Tyr 122 and Arg 121 from one monomer and His 80, Arg 32 and Lys 42 from the other monomer. We propose that this cluster forms the active site of the breakage-reunion reaction, with the other conserved positive charges (Arg 46 and Arg 47) anchoring the non-covalently bound $3^{\prime}$ end of the cleaved DNA.

We modelled a DNA duplex onto the GyrA59 structure by minimizing the distance between the tyrosines and the target phosphates, and by allowing the duplex to bend about a single 
a

E. coli DNA gyrase

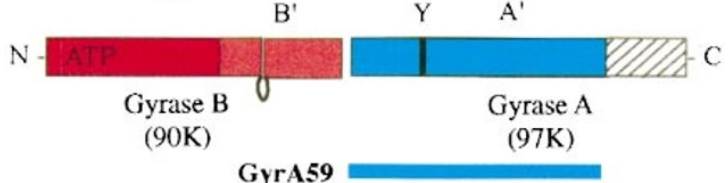

Topo II92
Figure $1 \mathbf{a}$, Domain organization of $E$. coli gyrase and yeast topoisomerase II. The yeast protein is a homodimer whereas the gyrase is a tetramer with two independent polypeptides. The domains perform similar functions, except for the C-terminal domains, which are not homologous ${ }^{21}$. b. Sequence alignment based on the three-dimensional structures. Secondary structure elements are defined by arrows ( $\beta$-strands) or bars ( $\alpha$-helices), and follow the nomenclature in ref. 3 , with additional elements primed. Lower-case letters indicate residues that have not been modelled. Residues that are homologous throughout the known type II topoisomerases ${ }^{6}$ are shaded.

Yeast topo II (164K)

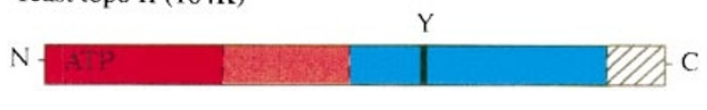

b

40

$\alpha .2$

${ }^{80} \quad \alpha 4$

GYrA msdl areitpvnieeel kssyldyamsviVGRA

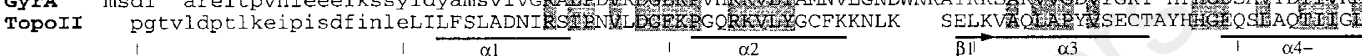

660

${ }_{700}^{1} \alpha 2$

140

$\beta 6$

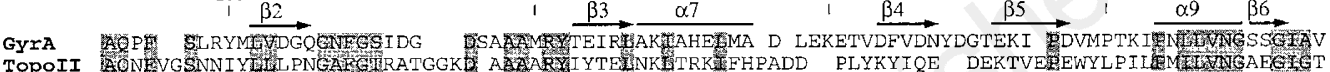

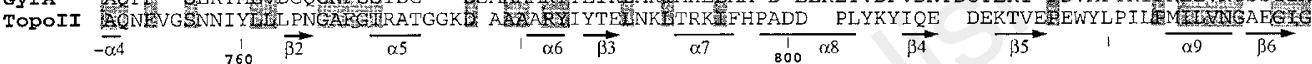

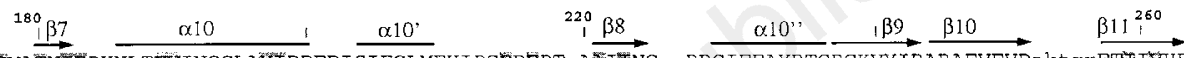

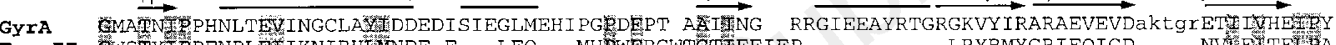

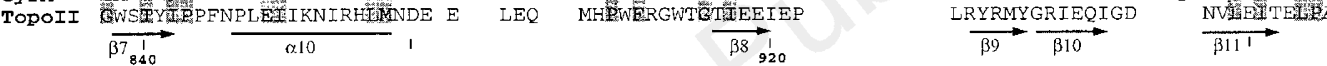

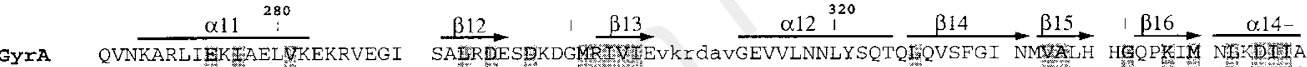

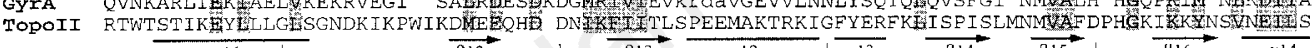

$\underset{\alpha 11 \underset{920}{1}}{\overrightarrow{\beta 12}}, \overrightarrow{\beta 13} \frac{\alpha_{\alpha 12}{ }_{960} \overrightarrow{\alpha 13}}{\overrightarrow{\beta 14}} \overrightarrow{\beta 15} \mid \overrightarrow{\beta 16} \quad \overrightarrow{\alpha 14}$
$\underset{360}{3}$
400
$\underline{\beta 17}$
$\stackrel{440}{1} \beta 18-$

GYIA AFVRHRREVVTRRTIFELRKARDRAHILEALAVASANI DPIIELIF RHAPTPAEAKTAFVANPWOLGNVAAMLERAGDDAARPEWLEPEFG

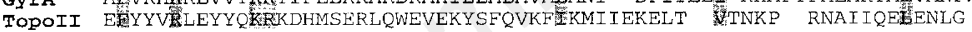
$\underset{\text { GYrA }}{\text { TOPOII }} \stackrel{-\beta 18}{\overrightarrow{\text { VRDGLYYLT }}}$

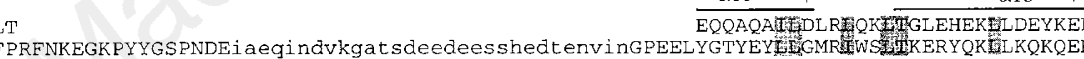

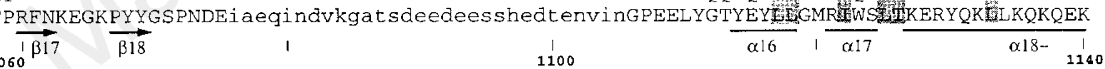

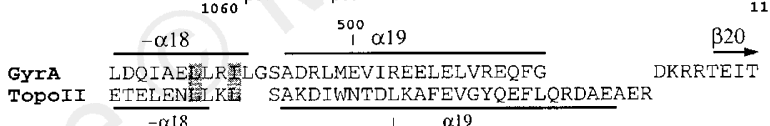

1040
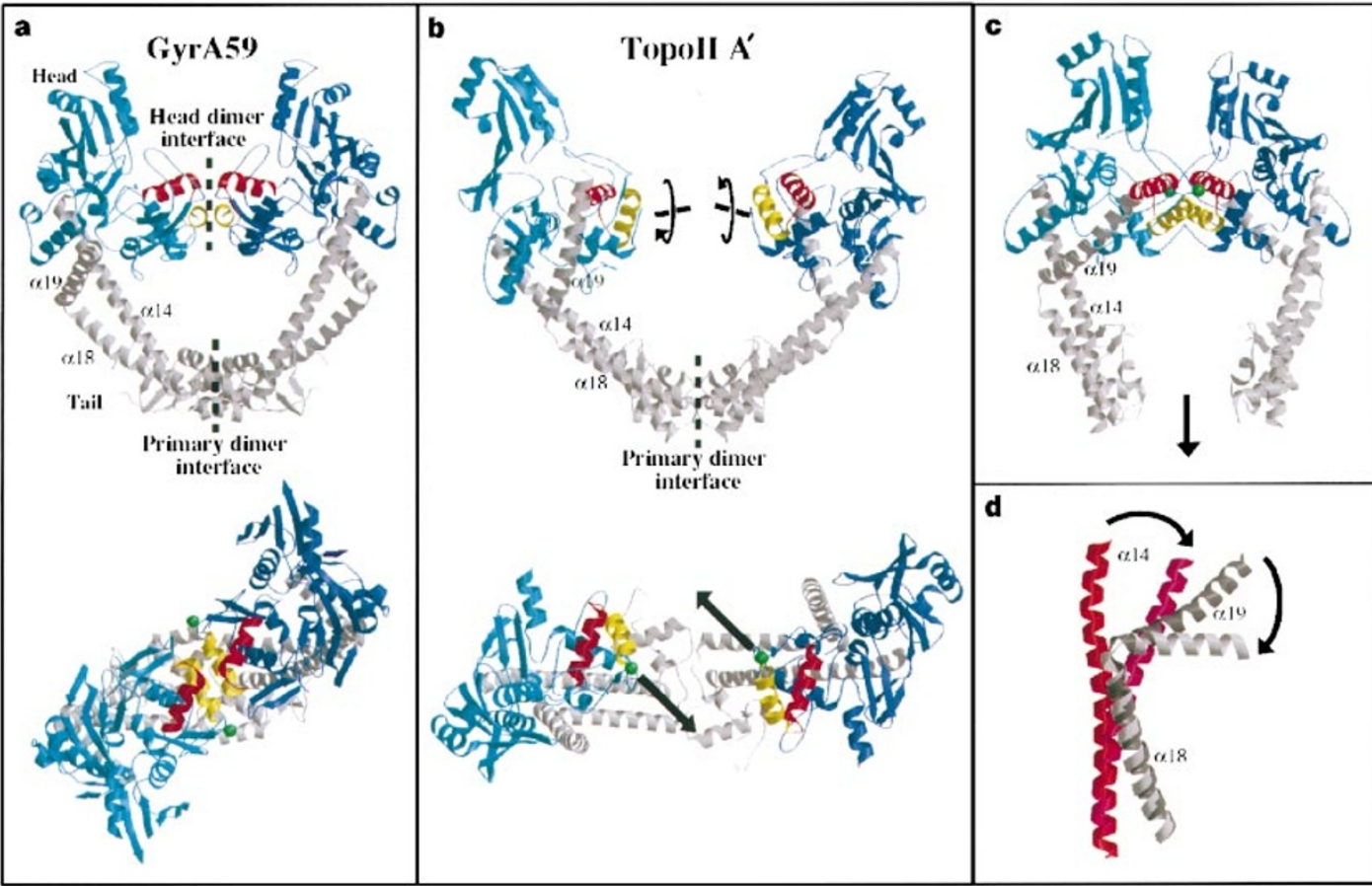

Figure 2 a, b, Orthogonal views of GyrA59 and the yeast topo II $A^{\prime}$ subunit. Head fragments are shown in blue, helix-turn-helix motifs in yellow $(\alpha 3)$ and red $(\alpha 4)$, tail domains in grey, active-site tyrosines as green spheres. Arrows show the direction of movement from topo II to gyrase. c, Opening of the 'second gate' in the primary dimer interface (see text). The $A^{\prime}$ subunits of yeast topo II were superimposed onto the GyrA59 dimer structure by least-squares fits onto the 'head' fragments. The view is rotated about a vertical axis to show the full extent of the gate $(\sim 22 \AA)$. d, Two conformations of the connecting helices, superimposed at the 'primary' dimer interface. Arrows show the direction of movement from topo II to gyrase. 
axis to follow the concave protein surface. This produced the following model (Fig. 3a): both 'recognition helices' (and most of the quinolone-resistance sites) lie in the central half turn of the DNA major groove; the duplex makes an angle of $\sim 20^{\circ}$ to the long axis of the dimer face, with the backbone phosphates from opposing strands lying in the basic grooves of the protein. Further proteinDNA contacts are made with the towers and with the $\beta 6-\beta 7$ hairpin, which contains a conserved exposed isoleucine side chain (Ile 174) that projects into the minor groove. The DNA curves upwards, towards the presumed location of the B-subunits, consistent with models of gyrase-DNA complexes derived from electron microscopy and neutron scattering ${ }^{7,8}$. This degree of DNA curvature (radius of curvature $\sim 70 \AA$ ) has been observed in other DNA-protein complexes 9 .
In our gyrase-DNA model, the target phosphoryl groups are still $7 \AA$ away from the tyrosines, so that either the DNA or the protein must adjust to bridge the gap. A rotation of the gyrase monomers that would bring the tyrosines closer to the phosphates is unlikely as this pushes the 'recognition' helices further apart. A substantial widening of the DNA major groove is a possibility, and this type of distortion has been observed in other DNA-protein complexes ${ }^{9,10}$. The quinolone drugs bind strongly to the gyrase-DNA complex but only weakly to protein or DNA alone ${ }^{11}$. The location of the quinolone-resistance mutations at the point where we predict that distortion of the DNA should occur (Fig. 3b, c) is consistent with the idea that the drugs interact with the protein and the DNA bases adjacent to the cleavage site.

The gyrase and yeast topo II structures provide complementary

\begin{tabular}{|c|c|c|c|c|c|c|}
\hline \multicolumn{7}{|c|}{ Table 1 Summary of crystallographic analysis } \\
\hline $\begin{array}{l}\text { Data set: } \\
\text { Wavelength }(\AA) \text { : }\end{array}$ & $\begin{array}{l}\text { Native } \\
1.54\end{array}$ & $\begin{array}{c}\mathrm{PtCl}_{4} \\
1.54\end{array}$ & $\begin{array}{l}\mathrm{PtCl}_{4} \\
1.067\end{array}$ & $\begin{array}{l}\text { PIP } \\
1.54\end{array}$ & $\begin{array}{l}\text { PIP } \\
0.87\end{array}$ & $\begin{array}{c}\text { MMN } \\
1.54\end{array}$ \\
\hline $\begin{array}{l}\text { Resolution }(\AA) \\
\text { Completeness }(\%) \\
\text { Redundancy } \\
R_{\text {sym }}(\%)^{*} \\
\text { No. of sites } \\
R_{\text {iso }}(\%) \dagger \\
R_{\text {cullis }}(\%) \neq \\
\text { Phasing power\$ } \\
\text { Overall figure of merit: }\end{array}$ & $\begin{array}{c}2.8 \\
100 \\
7 \\
4.7\end{array}$ & $\begin{array}{l}3.25 \\
98 \\
2 \\
7.4 \\
3 \\
17.6 \\
72 \\
1.6\end{array}$ & $\begin{array}{c}3.5 \\
92 \\
2 \\
12.6 \\
2 \\
15.4 \\
78 \\
1.3\end{array}$ & $\begin{array}{c}3.0 \\
100 \\
3 \\
7.5 \\
5 \\
11.5 \\
68 \\
1.8\end{array}$ & $\begin{array}{l}2.8 \\
84 \\
4 \\
7.9 \\
6 \\
14.0 \\
65 \\
1.9\end{array}$ & $\begin{array}{l}4.5 \\
97 \\
2 \\
7.3 \\
2 \\
10.9 \\
86 \\
0.9\end{array}$ \\
\hline \multicolumn{7}{|c|}{ Refinement statistics: } \\
\hline Resolution $(\AA)$ & $R$ factor $(\%) \|$ & $R_{\text {free }}(\%)$ & $\begin{array}{l}\text { r.m.s. bond } \\
\text { lengths }(\AA)\end{array}$ & $\begin{array}{l}\text { r.m.s. bond } \\
\text { angles }\end{array}$ & $\begin{array}{l}\text { No. of } \\
\text { atoms }\end{array}$ & $\begin{array}{l}\text { No. of } \\
\text { reflections }\end{array}$ \\
\hline $15-2.8$ & 22.6 & 31.0 & 0.006 & 1.13 & $3,774+15 \mathrm{H}_{2} \mathrm{O}$ & 16,367 \\
\hline
\end{tabular}

PIP, di- $\mu$-iodobis(ethylenediamine) diplatinum(II) nitrate; MMN, methylmercuric nitrate; $\mathrm{PtCl}_{4}$, potassium tetrachloroplatinate (II)

${ }^{*} R_{\mathrm{sym}}=\Sigma \Sigma j|\langle/\rangle-| j \mid / \Sigma\langle/\rangle$.

$\uparrow R_{\text {iso }}=\Sigma\left|F_{\mathrm{ph}}-F_{\mathrm{p}}\right| / \Sigma F_{\mathrm{p}}$.

$\ddagger R_{\text {cullis }}=\Sigma|| F_{\mathrm{ph}} \pm F_{\mathrm{p}}|-| F_{\mathrm{hc}}|| / \Sigma\left|F_{\mathrm{ph}} \pm F_{\mathrm{p}}\right|$

$\$$ Phasing power $=\langle F$ h $\rangle / E$

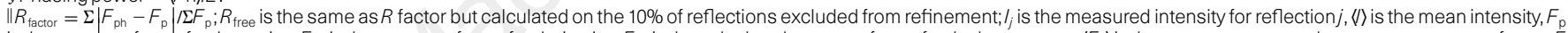

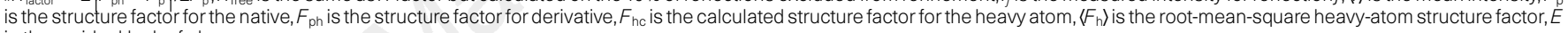
is the residual lack of closure.
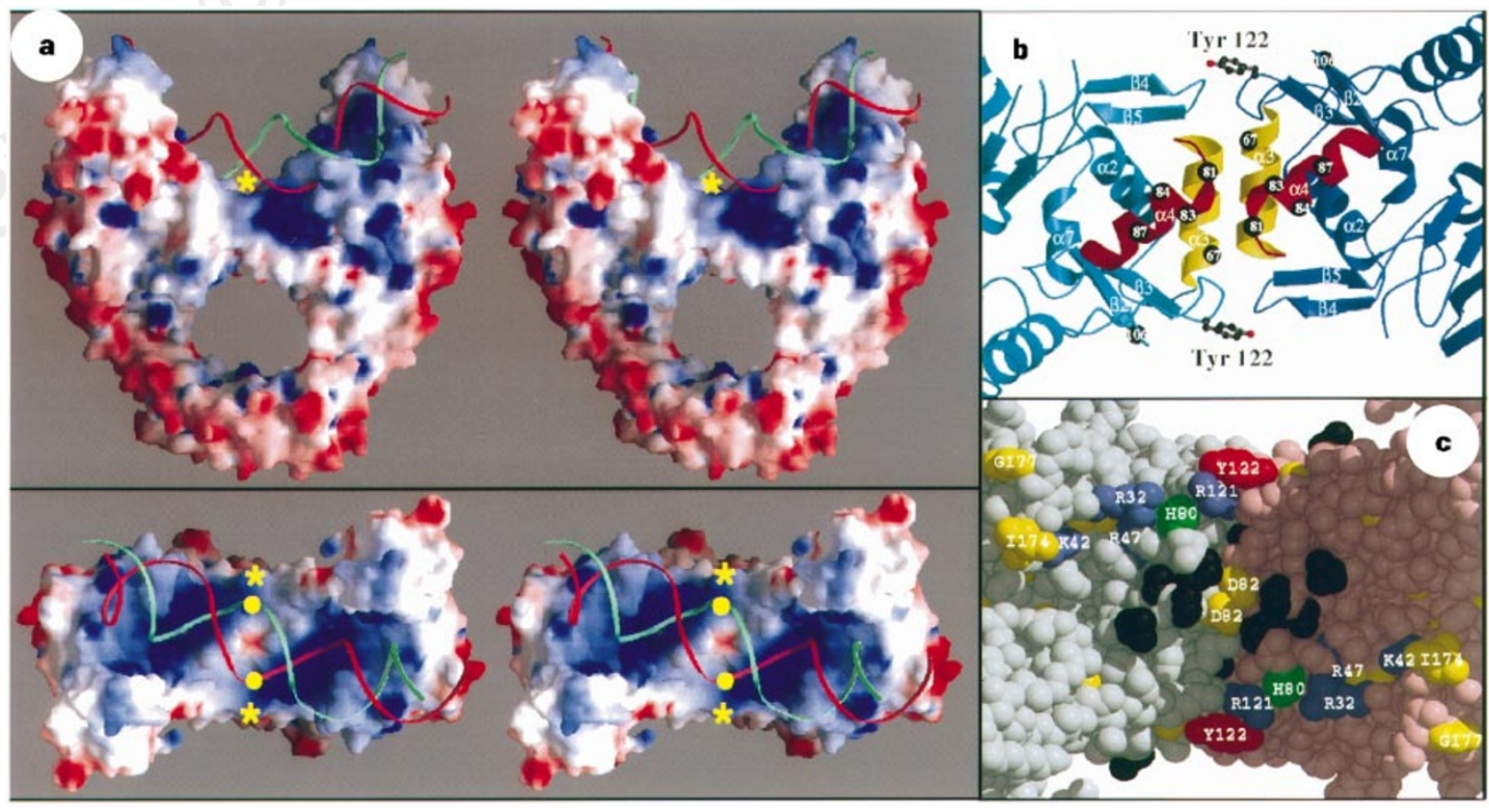

Figure 3 a, GRASP22 electrostatic surface potential of the GyrA59 dimer (orthogonal stereo views): negatively charged surfaces are in red, positively charged surfaces in blue. The DNA backbone is shown as a green and red ribbon; activesite tyrosines as yellow stars, target phosphoryl groups as yellow dots. b. Closeup of the 'head' dimer interface (colour scheme as in Fig. 2), with some secondary structure elements indicated. Quinolone-resistance sites are shown as numbered black spheres. c, Space-filling model, viewed as in b. Monomers are shown in pale green and pink. Quinolone-resistance sites are in black. Residues picked out in other colours are highly conserved within the topoisomerase family. 
pictures of the topoisomerase pathway. The gyrase structure shows how the $G$ segment can be held within the head fragments before strand passage, whereas the topo II structure shows how the T segment can pass through the active site. It has been proposed that after passing through the $\mathrm{G}$ segment, the T segment exits from the bottom of the molecule via the 'primary' dimer interface (the 'twogate' model $\left.{ }^{12,13}\right)$. The structures show how this might be done: the long connecting helices can exist in two distinct conformations, and if we impose the more extended hinge conformation observed in topo II onto the gyrase structure (Fig. 2c), we see that the primary dimer interface opens far enough to allow passage of the $\mathrm{T}$ segment.

\section{Methods}

Data collection and structure solution. GyrA59 (residues 2 to 523 of E. coli GyrA) was expressed and purified as described ${ }^{4}$. Crystals (space group $I 4_{1}$ : $a=119.6 \AA, c=95.0 \AA$ ) diffracting to $2.8 \AA$ resolution were obtained at $4{ }^{\circ} \mathrm{C}$ from $8 \%$ PEG8K, $20 \mathrm{mM} \mathrm{NaCl}, 0.1 \mathrm{M}$ Tris- $\mathrm{HCl}(\mathrm{pH} 8.5)$ after purification by crystallization under the same conditions (see Table 1). The crystals were flashfrozen under a stream of boiled-off nitrogen using $25 \%$ ethylene glycol as cryoprotectant. Heavy-atom soak conditions were: $1 \mathrm{mM}$ for $24 \mathrm{~h}\left(\mathrm{PtCl}_{4}\right)$; $4 \mathrm{mM}$ for $24 \mathrm{~h}$ (PIP); and $2 \mathrm{mM}$ for 4 weeks (MMN). The principal heavy-atom binding sites are: Cys $194(\mathrm{MMN})$; Met $120\left(\mathrm{PtCl}_{4}\right)$; Met 101, Met 178, Met 423, Met 499 and Glu 500 (PIP). Data sets were collected with an Raxis-II image plate on a Rigaku RU200HB generator or with a MAR image plate at DESY (Hamburg) and SRS (Daresbury). Data were processed with DENZO, SCALEPACK $^{14}$ and the CCP package ${ }^{15}$. The initial phases, produced by MLPHARE, were refined by solvent flattening and histogram matching with DM ( $60 \%$ solvent). The structure was solved by rounds of model building with the graphics display program $\mathrm{O}$ (ref. 16) (using the model of yeast topo II as a guide) and phase recombination.

Refinement and structural comparisons. Refinement consisted of rounds of simulated annealing and grouped $B$-factor refinement with bulk-solvent correction in X-PLOR (version 3.8) ${ }^{17}$. The present model consists of 477 residues; no convincing density is seen for the $\mathrm{N}$ terminus (residues 2-29) or the following surface loops: $251-256 ; 307-312$ and 426-429. The 'tower' domain is especially mobile (average main-chain $B$ value is $56 \AA^{2}$, compared with $27 \AA^{2}$ for the AP-like domain and $34 \AA^{2}$ for the tail and connecting helices), with several loops poorly defined. The stereochemistry is strongly restrained; there is one residue in a disallowed region of the Ramachandran plot; $88.5 \%$ lie in the most favourable regions. The head fragments of GyrA59 and topo II A' overlap with an r.m.s. deviation of $1.8 \AA$ for 251 homologous $\mathrm{C} \alpha$ atoms; the primary dimer interface overlaps with an r.m.s. deviation of $2.0 \AA$ for 37 homologous $\mathrm{C} \alpha$ atoms. Figures are drawn with MOLSCRIPT ${ }^{18}$, RASTER-3D ${ }^{19}$ and RENDER ${ }^{20}$.

Received 20 February; accepted 5 June 1997

1. Reece, R. J. \& Maxwell, A. DNA gyrase: structure and function. CRC Crit. Rev. Biochem. Mol. Biol. 26, 335-375 (1991).

2. Wigley, D. B. in Nucleic Acids and Molecular Biology (eds Eckstein, F. \& Lilley, D. M. J.) 165-176 (Springer, Berlin and Heidelberg, 1995)

3. Berger, J. M., Gamblin, S. J., Harrison, S. C. \& Wang, J. C. Structure at $2.7 \AA$ resolution of a $92 \mathrm{~K}$ yeast DNA topoisomerase II fragment. Nature 379, 225-232 (1996).

4. Reece, R. J. \& Maxwell, A. Probing the limits of the DNA breakage-reunion domain of the Escherichia coli DNA gyrase A protein. J. Biol. Chem. 266, 3540-3546 (1991).

5. Kampranis, S. C. \& Maxwell, A. Conversion of DNA gyrase into a conventional type II topoisomerase. Proc. Natl Acad. Sci. USA 93, 14416-14421 (1996).

6. Caron, P. R. \& Wang, J. C. Appendix II: Alignment of primary sequences of DNA topoisomerases. $A d v$ Pharmacol. 29B, 271-297 (1994).

7. Kirchhausen, T., Wang, J. C. \& Harrison, S. C. DNA gyrase and its complexes with DNA: direct observation by electron microscopy. Cell 41, 933-943 (1985).

8. Krueger, S. et al. Neutron and light-scattering studies of DNA gyrase and its complex with DNA. J. Mol. Biol. 211, 211-220 (1990).

9. Schultz, S. C., Shields, G. C. \& Steitz, T. A. Crystal structure of a CAP-DNA complex: the DNA is bent by $90^{\circ}$. Science $253,1001-1007$ (1991).

10. Somers, W. S. \& Phillips, S. E. V. Crystal structure of the met repressor-operator complex at $2.8 \AA$ resolution reveals recognition by $\beta$-strands. Nature 359, 387-393 (1992).

11. Willmott, C. J. R. \& Maxwell, A. A single point mutation in the DNA gyrase A protein greatly reduces the binding of fluoroquinolones to the gyrase-DNA complex. Antimicrob. Agents Chemother. 37, $126-$ 127 (1993).

12. Roca, J. \& Wang, J. C. DNA transport by a type II DNA topoisomerase: evidence in favor of a two-gate mechanism. Cell 77, 609-616 (1994).
13. Roca, J., Berger, J. M., Harrison, S. C. \& Wang, J. C. DNA transport by a type II topoisomerase: direct evidence for a two-gate mechanism. Proc. Natl Acad. Sci. USA 93, 4057-4062 (1996).

14. Otwinowski, Z. in Data Collection and Processing (eds Sawyer, L., Isaacs, N. \& Bailey, S.) 56-62 (SERC, Warrington, UK, 1993)

15. Collaborative Computational Project, N.4. The CCP4 suite: programs for protein crystallography. Acta Crystallogr. D 50, 760-763 (1994).

16. Jones, T. A. Interactive computer-graphics-FRODO. Meth. Enzymol. 115, 157-171 (1985).

17. Brünger, A. T. X-PLOR Version 3.1, a System for Crystallography and NMR. (New Haven, Connecticut, 1992).

18. Kraulis, P. J. Molscript-a program to produce both detailed and schematic plots of protein structures. J. Appl. Crystallog. 24, 946-950 (1991).

19. Merrit, E. A. \& Murphy, M. E. P. Raster3D version 2.0. A program for photorealistic molecular graphics. Acta Crystallogr. D 50, 869-873 (1994).

20. Bacon, D. J. \& Anderson, W. F. A fast algorithm for rendering space-filling molecule pictures. J. Mol. Graph. 6, 219-220 (1988)

21. Lindsley, J. E. \& Wang, J. C. On the coupling between ATP usage and DNA transport by yeast DNA topoisomerase II. J. Biol. Chem. 268, 8096-8104 (1993).

22. Nicholls, A. GRASP: Graphical representation and analysis of surface properties. (Columbia University, New York, 1992).

Acknowledgements. We thank C. Petosa, S. Kampranis, L. Bankston and M. Sutcliffe for discussion. We acknowledge the EMBL Hamburg outstation c/o DESY and the SRS in Daresbury for access to their faciities. This work was supported by the BBSRC. A.M. is a Lister Institute Jenner Fellow.

Correspondence and requests for materials should be addressed to R.C.L. (e-mail: rcl6@le.ac.uk). Coordinates have been deposited with the Protein Data Bank (1AB4).

\section{erratum}

\section{Radical fringe positions the apical ectodermal ridge at the dorsoventral boundary of the vertebrate limb}

\section{Concepción Rodriguez-Esteban, John W. R. Schwabe, Jennifer De La Peña, Bryon Foys, Brian Eshelman \& Juan Carlos Izpisúa Belmonte}

Nature 386, 360-366 (1997)

In Fig. 7 of this Article, the two arrows indicating signalling by $R$-fng at stage 15 were unclear; the correct figure is shown here.
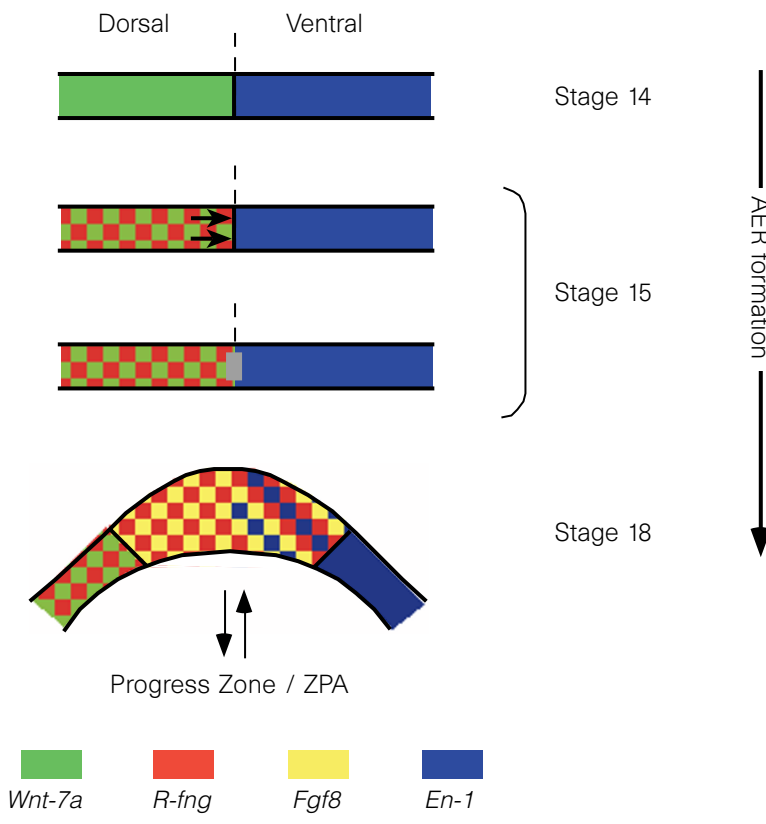